\title{
Espondiloptosis traumática C7-T1 sin compromiso neurológico
}

\author{
Traumatic spondyloptosis C7-T1 without neurologic deficit
Gómez-Flores G,* Gutiérrez-Herrera LE,* Dufoo-Olvera M,* Ladewig-Bernáldez GI,* Collado-Arce MGL,* Oropeza-Oropeza E,* López-Palacios JJ,* García-López O,* \\ May-Martínez ED,* Pérez-Jacobo G,* Silvas-Vásquez MR* \\ Hospital General «La Villa», Secretaría de Salud de la Ciudad de México.
}

RESUMEN. La espondiloptosis traumática es una lesión muy rara y grave generalmente causada por traumatismos de alta energía. Consiste en la dislocación anterior o posterior de $100 \%$ o más al cuerpo vertebral subyacente, lo que puede generar compresión y lesión total de la médula espinal, produciendo déficit neurológico; este tipo de lesión representa la etapa 4 y 5 de Allen. Caso clínico: Masculino de 50 años quien sufre accidente automovilístico al colisionar contra muro de contención, generándose lesión de tipo hiperextensión-compresión cervicotorácica, manejado con hemicorpectomía C7, discectomía C7-T1, espondilodesis con placa anterior (C6-C7, C7-T1), toma y aplicación de injerto, abordaje posterior + fascetectomías de C7 + fijación transfacetaria C6 y transpedicular de T1. Discusión: Encontramos que la estabilización temprana con pinza de Gardner más el abordaje anterior y posterior brindan adecuados resultados en cuanto a integridad sensitiva y motora del paciente así como una pronta rehabilitación.

Palabras clave: Espondiloptosis, traumática, cervical.

\section{Introducción}

La espondiloptosis traumática es una lesión muy rara y grave generalmente causada por traumatismos de alta energía, la cual consiste en la dislocación anterior o posterior de $100 \%$ o más al cuerpo vertebral subyacente, lo
ABSTRACT. Traumatic spondyloptosis is a serious injury usually caused by high-energy trauma; It consists of the anterior or posterior dislocation of $100 \%$ or more of the underlying vertebral body, which can become a total injury of the spinal cord, producing a neurological deficit; this type of injury represents stage 4 and 5 of Allen-Ferguson. Clinical case: A 50-year-old man who suffers a car accident, he receive frontal impact when he was a driver, colliding with the retaining wall, referred from another hospital to emergency room, managed with C7 hemicorpectomy, c7-t1 discectomy, spondylodesis with anterior plate (C6-T1), and posterior approach + Fascetectomies of C7-T1, facet joint screws C6 and transpedicular fixation of T1. Discussion: Subaxial cervical spondyloptosis is relatively rare clinical entity, a complete clinical examination is important in diagnosis, taking in considerations the injury mechanism. For treatment we have a multiple options, at this case anterior-posterior (360 degrees) treatment it was the better option for Us; however, must be personalized and consider the early rehabilitation of patient.

Keywords: Spondyloptosis, traumatic, cervical.

* Clínica de Columna de la Secretaría de Salud de la Ciudad de México. Hospital General «La Villa».

Correspondencia:

Gerson Gómez Flores

Av. San Juan de Aragón Núm. 285, Col. Granjas, Alcaldía Gustavo A Madero, C.P. 07460, CDMX.

E-mail: drgersongomez@gmail.com

Citar como: Gómez-Flores G, Gutiérrez-Herrera LE, Dufoo-Olvera M, Ladewig-Bernáldez GI, Collado-Arce MGL, Oropeza-Oropeza E, et al. Espondiloptosis traumática C7-T1 sin compromiso neurológico. Acta Ortop Mex. 2020; 34(6): 412-416. https://dx.doi.org/10.35366/99140

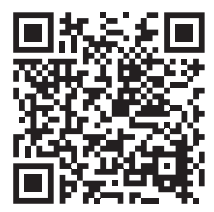


Entre las lesiones más severas secundarias al trauma se encuentran las que comprometen a la médula espinal y a la columna vertebral. Por su etiología la mayoría de éstas cursan con patologías que aumentan su morbilidad y mortalidad. Cada año en los Estados Unidos se presentan aproximadamente 150,000 lesiones en la columna vertebral. . $3,3,4^{3}$ Aproximadamente $75 \%$ de las lesiones cervicales ocurren en la columna C3-C7. ${ }^{6}$

Entre la literatura médica se menciona al género masculino como el más susceptible a este tipo de lesiones, con una edad promedio que va desde 20 hasta 50 años de edad. Las mujeres representan $40 \%$ con dos picos de edad, el primero de 20 a 50 años y el segundo de 60 a 80 años. Entre las causas más comunes se encuentran caídas de alta energía y los accidentes de tráfico en orden de frecuencia: automóvil, motocicleta, bicicleta y peatones.?

La lesión de la columna cervical se observa en 3.7\% de los pacientes que presentan lesiones a columna vertebral, siendo $41.9 \%$ inestables. ${ }^{7,8,9}$ La lesión cerebral se asocia con una incidencia de $5.4 \%$ a lesión de columna cervical. En comparación con otras regiones, las fracturas cervicales se asocian con mayor riesgo de déficit neurológico y lesión completa de la médula espinal.

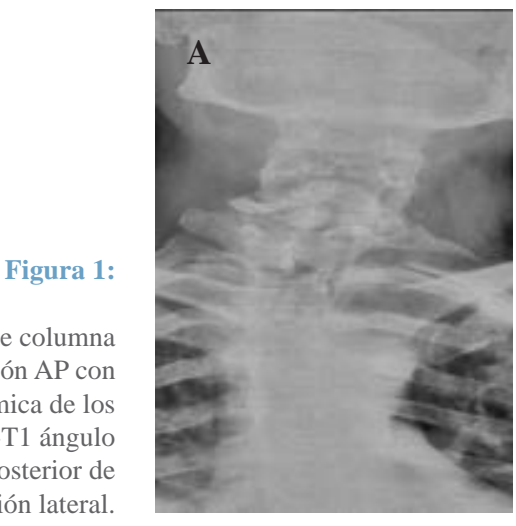

Radiografías simples de columna cervical. A y B) Proyección AP con incongruencia anatómica de los cuerpos vertebrales de C7-T1 ángulo de lateralización anteroposterior de $13.7^{\circ}$. C) Proyección lateral.

\section{Caso clínico}

Masculino de 50 años, originario y residente de la Ciudad de México (peso $74 \mathrm{~kg}$ ) quien sufre accidente automovilístico; colisiona contra muro de contención produciéndose desaceleración frontal que genera lesión de tipo hiperextensióncompresión de la columna cervicotorácica. Refirió desde el momento del accidente dolor intenso en cuello al realizar movimientos. Presentó pérdida de conciencia; fue llevado por paramédicos al servicio de urgencias más cercano donde se inmovilizó con collarín cervical rígido. Posteriormente trasladado a otro hospital donde se realizan estudios encontrando lesión cervical, se inicia esquema NASCIS II y es trasladado a nuestra unidad clínica de columna para manejo y tratamiento.

Durante la evaluación en nuestro servicio de urgencias, encontramos paciente desubicado por estado etílico. Craneofaciales con presencia de laceraciones superficiales a nivel frontal, hematoma periorbitario bilateral (signo de mapache) y subconjuntival, pupilas isocóricas normorrefléxicas. Exploración de nervios craneales: I no valorado, II fondo de ojo sin papiledema, III, IV, VI pupilas simétricas $3 \mathrm{~mm}$, reflejo fotomotor y consensual presentes, nistagmo ausente, $\mathrm{V}$ sensibilidad normal, reflejo corneal
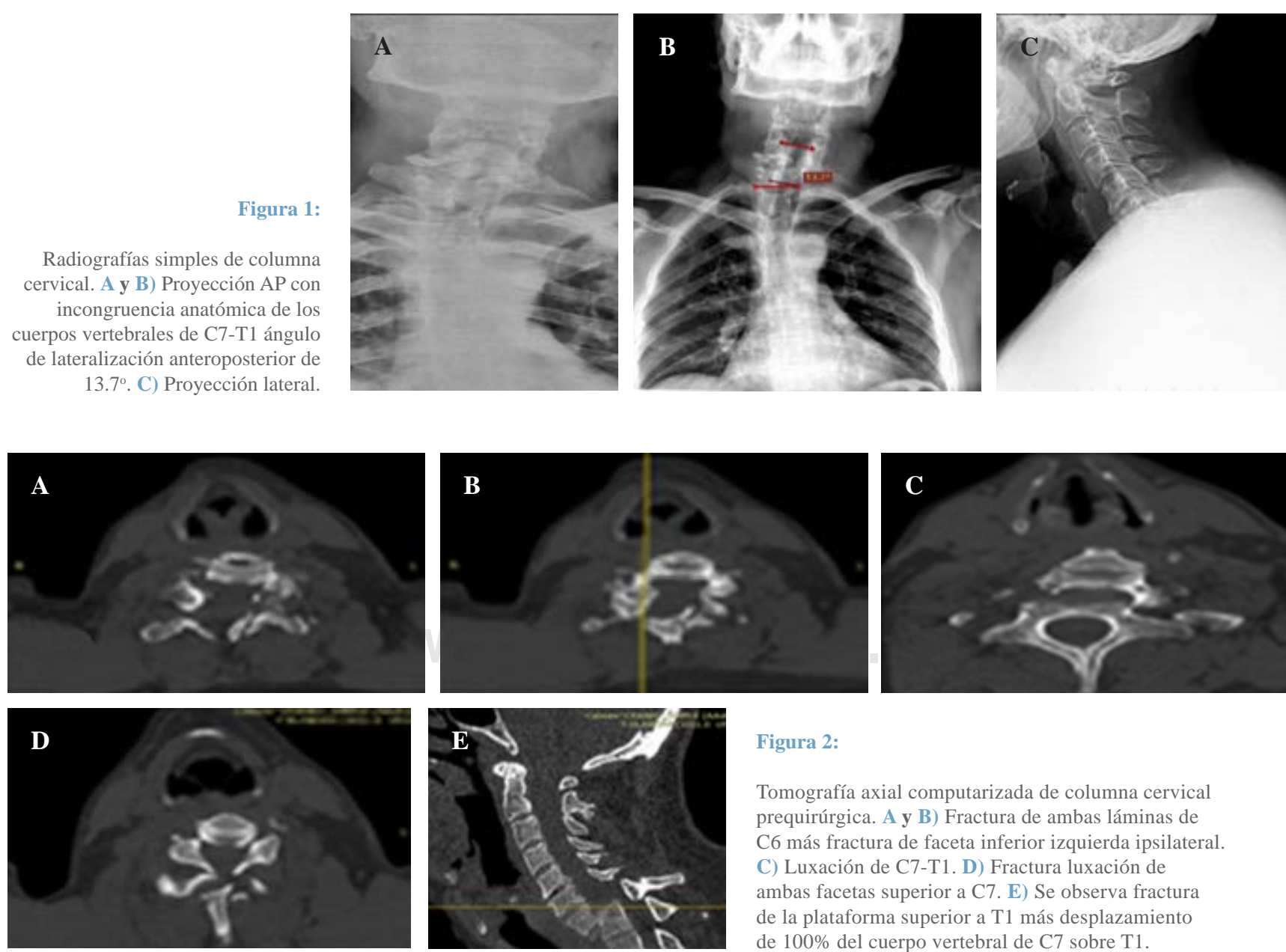

Figura 2:

Tomografía axial computarizada de columna cervical prequirúrgica. A y B) Fractura de ambas láminas de C6 más fractura de faceta inferior izquierda ipsilateral. C) Luxación de C7-T1. D) Fractura luxación de ambas facetas superior a C7. E) Se observa fractura de la plataforma superior a T1 más desplazamiento de $100 \%$ del cuerpo vertebral de C7 sobre T1. 

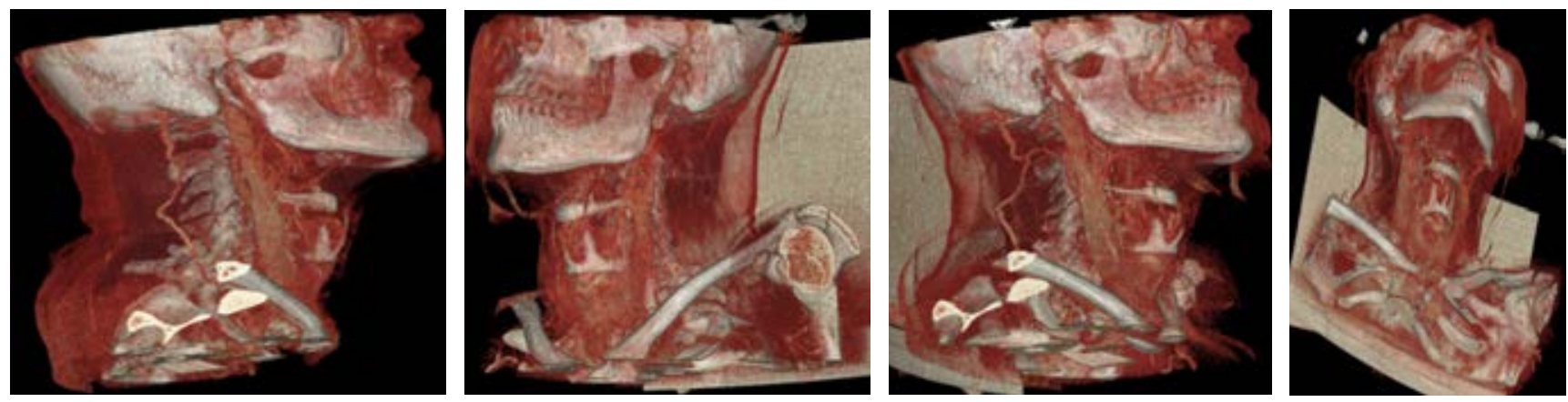

Figura 3: Angiotomografía prequirúrgica de vasos supraaórticos permite apreciar vértebras, carótidas, subclavias, tronco braquicefálico y arco aórtico.
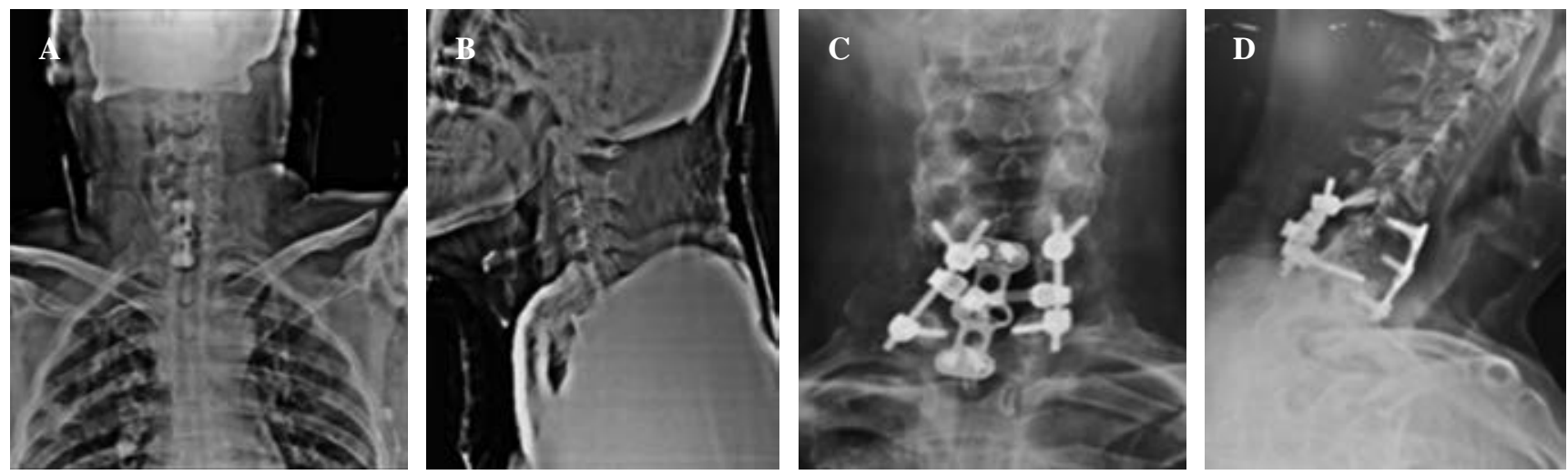

Figura 4: Radiografías postquirúrgicas. A) Rx anteroposterior cervical. B) Rx lateral de columna cervical. Con placa cervical anterior de dos niveles. C y D) Fijación facetaria de C6 más fijación transpedicular de T1.

conservado, apertura y fuerza de cierre de la boca sin alteraciones, VII mímica facial conservada, sensibilidad gustativa conservada, VIII coclear y vestibular normales, IX sensibilidad gustativa sin alteraciones, deglución conservada, X velo paladar con elevación simétrica, voz normal, XI simetría de hombros y escápulas, contracción esternocleidomastoideo y trapecio conservada, XII sin alteraciones. Función cerebelosa: no valorable, meníngeos: ausentes, Babinski y sucedáneos ausentes.

Cuello con dolor a la palpación, sensibilidad normal, tórax sin crepitaciones, con sensibilidad de T2 a T12, reflejos presentes a nivel de T7 a T12; pelvis sin datos de inestabilidad al realizar maniobras de Erichsen y Volkman. Se encuentra tono y automatismo anal normales, reflejo bulbo cavernoso presente.

Extremidades superiores: izquierda con presencia de deformidad en tercio medio de brazo así como equimosis en cara lateral del mismo y presencia de edema $(++)$, se palpaba crepitación al mismo nivel; encontramos C5 a C8 con sensibilidad presente, a la exploración motora: arcos de movilidad disminuidos por dolor, muestra $3 / 5$ en escala de Lovett, ROTS (++++), pulsos periféricos normales, llenado capilar dos segundos. Derecha sin alteraciones, nivel sensitivo C5 a C8, a la exploración motora: C5 a C8, 5/5. ROTS presentes, llenado capilar de dos segundos. Extremidades inferiores ambas normales a la palpación, sensibi- lidad presente 12 a S3, a la exploración motora: T12 a S1, $5 / 5$, ROTS (++++), llenado capilar de dos segundos.

Se realiza serie traumática encontrando en la proyección AP de columna cervical (Figura 1A y B) incongruencia anatómica con un ángulo de lateralidad marcado (Figura 1C). Proyección lateral C1-C6 (Figura 2). TAC cráneo fractura de ambas láminas de C6 fractura de faceta inferior izquierda ipsilateral (Figura 2A y B) luxación de C7 sobre T1 (Figura $2 C)$. Fractura de la plataforma superior de T1 más desplazamiento de $100 \%$ del cuerpo vertebral de C7 sobre el cuerpo vertebral de T1 (Figura 2C y D). Angiotomografía (Figura 3) realizada permitió descartar lesiones en vasos supraaórticos y descartar variantes que complicaran la realización del abordaje.

\section{Tratamiento}

A su ingreso se maneja con protocolo universal para paciente politraumatizado; se coloca pinza de Gardner-Wells sólo para estabilización craneal.

Se programa primer tiempo quirúrgico para realizar hemicorpectomía C7, discectomía C7-T1, espondilodesis con placa anterior dos niveles C6-C7, C7-T1, más toma y aplicación de injerto de cresta ilíaca, se corrobora resultado mediante TAC. Se realiza segundo tiempo quirúrgico siete días posteriores de primera intervención realizando aborda- 
je cervical posterior + fascetectomías de C7 + fijación transfacetarias C6 y transpedicular de T1 con tornillos, barras y un cross-link (Figura 4).

La fractura de base anterior de cráneo se manejó de forma conservadora por no contar con criterios quirúrgicos ni contraindicaciones para colocar tracción cefálica, la fractura de húmero se inmovilizó con férulas para posteriormente ser manejado por el servicio de ortopedia.

\section{Discusión}

La espondiloptosis se trata de una fractura bilateral del arco vertebral con desplazamiento anterior del cuerpo vertebral de $100 \%$. La falla ligamentosa se produce en dos niveles, posteriormente entre la vértebra suprayacente y fracturada y en la parte anterior entre la vértebra fracturada y la fractura subyacente. ${ }^{1}$ Nuestro caso fue una espondiloptosis traumática reciente, realizamos colocación de pinza de Gardner-Wells y maniobras de reducción preoperatoriamente de manera temporal, ${ }^{10}$ lo que permitió la estabilización para prevenir movilidad en la región de la fractura, considerando que este tipo de pacientes necesitan de movilidad controlada para evitar complicaciones como úlceras por decúbito e infecciones nosocomiales; del mismo modo la pinza facilitó el traslado a la sala quirúrgica y la movilización durante el procedimiento quirúrgico. El uso de la tracción con pinza de Garde debe ser juicioso y supervisado por fluoroscopía o radiografías seriadas, tal como lo mencionan García López y colaboradores, ${ }^{11}$ ya que la espondiloptosis es una lesión ligamentosa de tres columnas y la sobredistracción puede provocar fácilmente mayor daño medular, por lo que con nuestro paciente nunca superamos el peso correspondiente al cráneo y nivel cervical para evitar sobredistracción. ${ }^{12}$ Realizamos tracción pese a la fractura lineal no desplazada del piso anterior valorando el riesgo beneficio que otorgaría al paciente; sin embargo, en los casos con fracturas temporoparietales es importante intentar la reducción inicial cerrada con el paciente despierto ante el riesgo de hundimiento de la fractura y/o lesión intracraneal a parénquima o desarrollar hematomas epidurales.

La toma de decisiones con respecto al manejo por vía anterior versus vía posterior de una luxación cervical es relevante; ; ${ }^{1,13,14,15}$ para nuestro paciente se decidió realizar el primer tiempo quirúrgico por vía anterior para retirar completamente el disco afectado y de esta forma evitar la compresión medular con el mismo antes de realizar el abordaje posterior. El objetivo final de cualquiera de los procedimientos es obtener una reducción anatómica, descompresión medular y evitar inestabilidad con una fusión adecuada. Los tratamientos informados de la espondiloptosis han incluido abordaje anterior con o sin instrumentación y fusión posterior y anterior. Se planificó estabilización más fijación anterior y fusión en el primer tiempo quirúrgico. En la siguiente etapa se programó la instrumentación posterior y la fusión para lograr la estabilización. Se logró la reducción total confirmándose con Rx y TAC de control con una evolución clínica (Figura 5) satisfactoria del paciente.

En los diferentes reportes encontrados en la bibliografía ${ }^{16}$ hay una gran variedad de mecanismos de lesiones y presentaciones clínicas ${ }^{17}$ las cuales hay que analizar de manera consciente aunado a un protocolo bien definido de manejo que asegure la integridad neurológica del paciente en aqué-
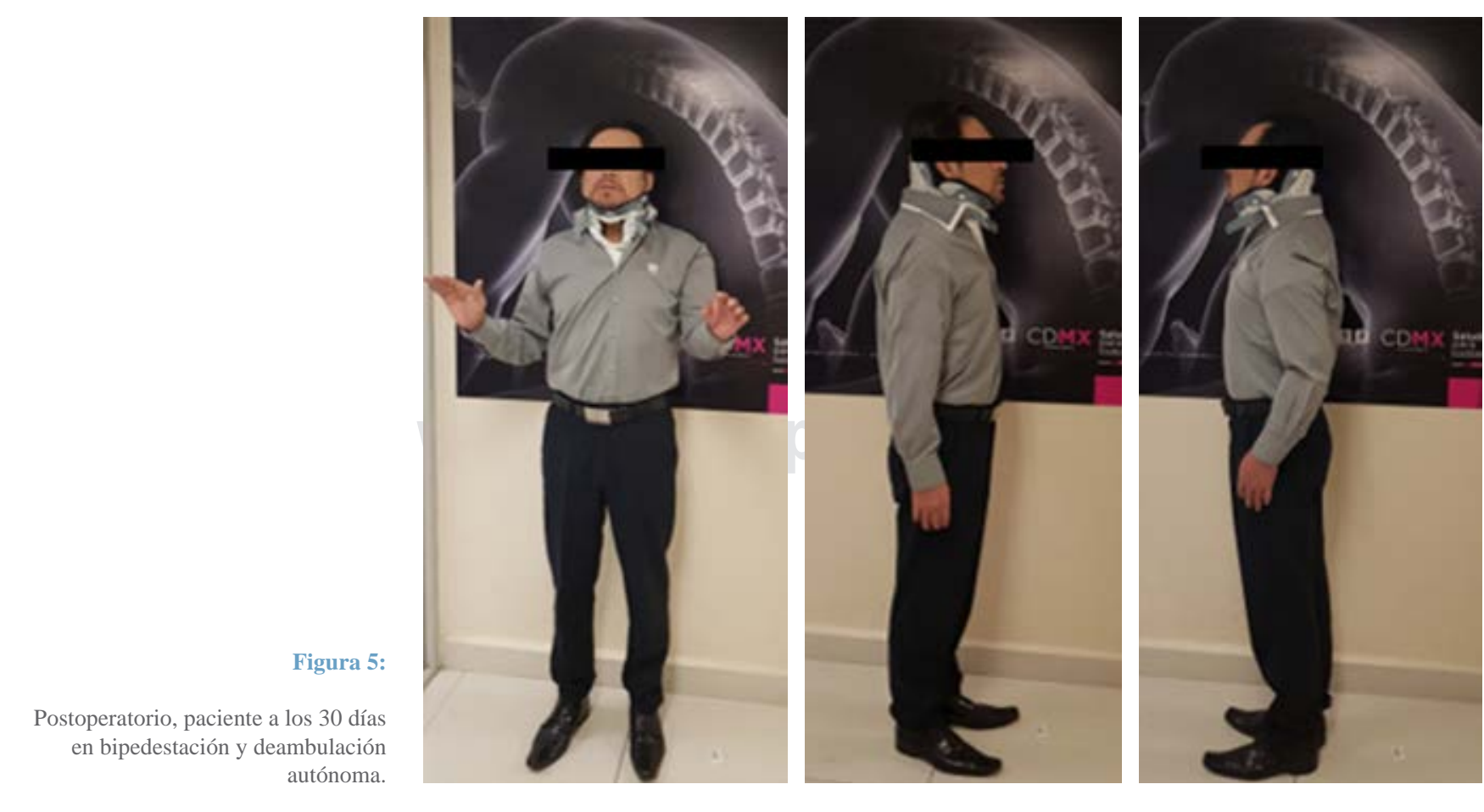
llos que la preservan, la mejoría neurológica en aquéllos con posibilidad y la estabilización adecuada para prevenir comorbilidades futuras.

Referencias

1. Alle BL Jr, Ferguson RL, Lehmann TR. A mechanistic classification of closed, indirect fractures and dislocation of the lower cervical spine. Spine (Phila Pa 1976). 1982; 7(1): 1-27.

2. Hadley MN, Walters BC, Grabb PA. Guidelines for the management of cervical Spine and spinal cord injuries. Clin Neurosurg. 2002; 49: 407-98.

3. Lowery DM, Wald MM, Browne BJ. Epidemiology of cervical spine injury victims. Ann Emerg Med. 2001; 38(1): 12-6.

4. Kwon BK, Vaccaro AR, Grauer JN. Sub-axial cervical spine trauma. $J$ Am Acad Orthop Surg. 2006; 14(2): 78-89.

5. Hadley MN, Walters BC, Grabb PA. Guidelines for management of acute cervical injuries. Neurosurgery. 2002; 50 (Suppl): 1-5.

6. Casper DS, McKenzie J, Schroeder GD. Controversial cervical spine fractures: classification and treatment. Seminars in Spine Surgery. 2017; 29(1): 41-9.

7. Leucht P, Fischer K, Muhr G, Mueller EJ. Epidemiology of traumatic spine fractures. Injury. 2009; 40: 166-72.

8. Sekhon LH, Fehlings MG. Epidemiology, demographics and pathophysiology of acute spinal cord injury. Spine (Phila Pa 1976). 2001; 26(24 Suppl.): S2-12.
9. Shekhar H, Khan S. Cervical spine injuries. Orthopaedisc and Trauma. 2016; 30(5): 390-401.

10. Hadley MN, Walters BC, Grabb BC. Initial closed reduction of cervical spine fracture: dislocation injuries. Neurosurgery. 2002; 50(3 Suppl.): S44-50.

11. Garcia-López OF. Luxación cervical mediante reducción cerrada segmento inferior (C-3 aC-7). Ortho-tips. 2007; 3(3): 188-93.

12. Vaccaro AR, Falatyn SP, Flanders AE. Magnetic resonance evaluation of the intervertebral disc, spinal ligaments and spinal cord before and after closed traction reduction of cervical spine dislocations. Spine (PhilaPa 1976). 1999; 24(12): 1210-7.

13. Shah KC, Raishekhar V. Successful management of posttraumatic c7T1 spondyloptosis with uninstrumented ventral surgery. Surg Neurol. 2004; 62(5): 431-4.

14. Muzumdar DP, Goel A. $\mathrm{C}_{2}$ over $\mathrm{C}_{3}$ spondyloptosis in a case with absent posterior elements. Report of an unusual case and analysis of treatment options. J Clin Neurosci. 2004; 11(6): 675-7.

15. Wiseman DB, Bellabarba C, Mirza SK. Anterior versus posterior surgical treatment for traumatic cervical spine dislocation. Curr Opin Orthop. 2003; 14(3): 174-81.

16. McConell We, Howard PR, Guzman HM. Analysis of human test Kinematic responses to low velocity rear end impacts. In: Vehicle and occupant kinematics: simulation and modeling (SP-97). Detroit. Warrendale, PA: Society for Automotive Engineering, 1993-21-30. SAE Technical Paper Series 930889.

17. Kirshblum S, Burns S, Waring W. International standards for neurologia classification of spinal cord injury (revised 2011). J Spinal Cord Med. 2011; 34(6): 535-46. 